\title{
Zum Verhältnis des nationalen Rechts zum Europarecht nach der Rechtsprechung des Bundesverfassungsgerichts
}

\author{
Hans-Jürgen PAPIER
}

\section{Einführung}

Die Anwendung europäischen Rechts ist aus unserer täglichen Rechtspraxis kaum hinweg zu denken. In der Konsequenz stellen sich vielfältige Fragen des Verhältnisses von nationalem Recht zum Europarecht. Zu dem Symposium „50 Jahre EU - 50 Jahre Rechtsprechung des Europäischen Gerichtshofs zum Arbeits- und Sozialrecht" haben Sie mich eingeladen, um dieses Thema aus der Sicht des Bundesverfassungsgerichts und damit des deutschen Verfassungsrechts - zu beleuchten. Dies tue ich sehr gern. Wegen der nur begrenzt zur Verfügung stehenden Zeit muss ich mich allerdings auf eine knappe Darstellung aus der Sicht des Bundesverfassungsgerichts besonders wichtiger Fragestellungen beschränken. Hier rückt zum einen der Grundrechtsschutz gegen sekundäres Gemeinschaftsrecht und zum anderen die Kontrolle der Kompetenzgrenzen der Gemeinschaft in den Blick.

\section{Grundrechtsschutz gegen sekundäres Gemeinschaftsrecht}

Lassen Sie mich zunächst in groben Strichen die Entwicklung der Rechtsprechung des Bundesverfassungsgerichts zu den Problemen des Grundrechtsschutzes nachzeichnen. Den Ausgangspunkt bildete der Solange I-Beschluss aus dem Jahre 19741. Bereits zehn Jahre zuvor, in der Rechtssache Costa/E.N.E.L. ${ }^{2}$, hatte der EuGH die Grundsätze der unmittelbaren Geltung und des Vorrangs des Gemeinschaftsrechts als einer eigenständigen Rechtsordnung entwickelt; erst in Ansätzen erkennbar war auf der anderen Seite die Herausbildung eines begrenzenden Gegengewichts zur Rechtsetzung der Gemeinschaft, insbesondere durch grundrechtliche Gewährleistungen. Vor diesem Hintergrund hat sich das Bundesverfassungsgericht in der Solange I-Entscheidung die umfassende Prüfung von Gemeinschaftsrecht am Maßstab der Grundrechte des Grundgesetzes vorbehalten, „solange der Integrationsprozess der Gemeinschaft nicht so weit fortgeschritten ist, dass das Gemeinschaftsrecht auch einen von einem Parlament beschlosse-

1 BVerfGE 37, S. $271 \mathrm{ff}$.

2 EuGH, Rs. 6/64, Slg. 1964, 1251. 
nen und in Geltung stehenden formulierten Katalog von Grundrechten enthält, der dem Grundrechtskatalog des Grundgesetzes adäquat ist" ${ }^{\text {"3 }}$.

Nachdem das Bundesverfassungsgericht im sog. Vielleicht-Beschluss aus dem Jahre $1979^{4}$ eine Überprüfung dieses Vorbehalts in Aussicht gestellt hatte, stellte es dann in dem Solange II-Beschluss von $1986^{5}$ fest, dass ,mittlerweile im Hoheitsbereich der Europäischen Gemeinschaft ein $\mathrm{Ma} \beta$ an Grundrechtsschutz erwachsen ist, das nach Konzeption, Inhalt und Wirkungsweise dem Grundrechtsstandard des Grundgesetzes im wesentlichen gleichzuachten ist" 6 . Als Konsequenz kehrte das Bundesverfassungsgericht seine „Solange-Formel“ um. „Solange“, so heißt es nunmehr, „die Europäischen Gemeinschaften [...] einen wirksamen Schutz der Grundrechte gegenüber der Hoheitsgewalt der Gemeinschaften generell gewährleisten, der dem vom Grundgesetz als unabdingbar gebotenen Grundrechtsschutz im wesentlichen gleichzuachten ist, zumal den Wesensgehalt der Grundrechte generell verbürgt, wird das Bundesverfassungsgericht seine Gerichtsbarkeit über die Anwendbarkeit von abgeleitetem Gemeinschaftsrecht [...] nicht mehr ausüben und dieses Recht mithin nicht mehr am Maßstab der Grundrechte des Grundgesetzes überprüfen"7.

Sieben Jahre später, im Maastricht-Urteil von $1993^{8}$, hat das Bundesverfassungsgericht seine Rechtsprechung nochmals zusammengefasst und um eine weitere Formel ergänzt. Das Bundesverfassungsgericht übe, so heißt es in der Entscheidung, „,seine Gerichtsbarkeit über die Anwendbarkeit von abgeleitetem Gemeinschaftsrecht in Deutschland in einem „Kooperationsverhältnis“ zum Europäischen Gerichtshof aus, in dem der Europäische Gerichtshof den Grundrechtsschutz in jedem Einzelfall für das gesamte Gebiet der Europäischen Gemeinschaften garantiert, das Bundesverfassungsgericht sich deshalb auf eine generelle Gewährleistung der unabdingbaren Grundrechtsstandards beschränken kann“"9.

Die prozessrechtlichen Konsequenzen wurden in dem Beschluss zur Bananenmarktordnung vom Juni $2000^{10}$ verdeutlicht. Das Bundesverfassungsgericht präzisierte hier, dass Verfassungsbeschwerden und Vorlagen von Gerichten unzulässig sind, ,wenn ihre Begründung nicht darlegt, dass die europäische Rechtsentwicklung einschließlich der Rechtsprechung des Europäischen Gerichtshofs nach Ergehen der Solange IIEntscheidung unter den erforderlichen Grundrechtsstandard abgesunken sei“"11.

\footnotetext{
3 BVerfGE 37, S. 271 (285).

4 BVerfGE 52, S. $187 \mathrm{ff}$.

5 BVerfGE 73, S. 339ff.

6 BVerfGE 73, S. 339 (378).

7 BVerfGE 73, S. 339 (387).

8 BVerfGE 89, S. $155 \mathrm{ff}$.

9 BVerfGE 89, S. 155 (175).

10 BVerfGE 102, S. 147ff.

11 BVerfGE 102, S. 147 (164).
} 
Hinweisen will ich schließlich auch auf einen aktuellen Beschluss des Ersten Senats des Bundesverfassungsgerichts vom 13. März $2007^{12}$ zum Thema Emissionshandel. In diesem Beschluss hat der Senat ausgeführt - übrigens ähnlich wie der französische Conseil d'État in seiner Entscheidung vom 8. Februar $2007^{13}$ in einem vergleichbaren französischen Fall -, dass auch innerstaatliche Rechtsnormen, mit denen Richtlinien des Gemeinschaftsrechts umgesetzt werden, die den Mitgliedstaaten keinen Umsetzungsspielraum belassen, sondern zwingende Vorgaben machen, vom Bundesverfassungsgericht und den Fachgerichten nicht am Maßstab der Grundrechte des Grundgesetzes gemessen werden, solange auf Gemeinschaftsebene der erforderliche Grundrechtsschutz gewährleistet ist. Zur Gewährung effektiven Rechtsschutzes sind die Fachgerichte verpflichtet, solche gemeinschaftsrechtlichen Vorgaben an den Gemeinschaftsgrundrechten zu messen und gegebenenfalls ein Vorabentscheidungsverfahren nach Art. 234 EG durchzuführen. Nach allem ist - so meine ich - ein modus vivendi zwischen Bundesverfassungsgericht und Europäischem Gerichtshof gefunden, der akute Konflikte in Grundrechtsfragen praktisch ausschließt.

\section{Kontrolle der Kompetenzgrenzen der Gemeinschaft}

Damit noch nicht geklärt ist allerdings die Problematik, die sich daraus ergibt, dass sich das Bundesverfassungsgericht nach wie vor eine prinzipielle Prüfungskompetenz über das Gemeinschaftsrecht vorbehalten und diese lediglich zurückgestellt hat. Eine solche Prüfungskompetenz ist ohne weiteres gegeben, soweit es um das deutsche $\mathrm{Zu}$ stimmungsgesetz zur vertraglichen Begründung der Union oder zu Änderungen ihrer vertraglichen Grundlagen und um die damit verbundene Übertragung von Hoheitsrechten geht. Das Bundesverfassungsgericht hat im Maastricht-Urteil ausgesprochen, dass der Übertragung von Hoheitsrechten auf die Europäische Union Grenzen gesetzt seien und dass die übertragenen Rechte und das beabsichtigte Integrationsprogramm hinreichend bestimmbar festzulegen seien. Durch die Übertragung von Aufgaben und Befugnissen auf die Union dürfen die Entscheidungs- und Kontrollzuständigkeiten des Deutschen Bundestags nicht in einer Weise entleert werden, die das Demokratieprinzip in seinem unantastbaren Kern (Art. 79 Abs. 3 GG) verletzt. Entscheidend sei ferner, dass die Mitgliedschaft der Bundesrepublik und die sich daraus ergebenden Rechte und Pflichten für den Gesetzgeber voraussehbar im Vertrag umschrieben und durch ihn im Zustimmungsgesetz hinreichend bestimmbar normiert worden seien. Das Erfordernis hinreichender Bestimmtheit der eingeräumten Hoheitsrechte wäre insbesondere dann verletzt, wenn eine Kompetenz-Kompetenz der Europäischen Union begründet würde,

$121 \mathrm{BvF} 1 / 05$.

$13 \mathrm{CE} \mathrm{N}^{\circ} 287110$. 
wenn sich - mit anderen Worten - die Europäische Union selbst und ohne vorherige Vertragsänderung weitere Aufgaben und Befugnisse verschaffen könnte ${ }^{14}$.

Ist das Integrationsprogramm einmal verbindlich festgelegt, so geht es darum, dass dieses Programm und dessen Grenzen in der Praxis tatsächlich eingehalten werden. Auch Art. I-6 des Entwurfs eines Europäischen Verfassungsvertrags spricht diese Selbstverständlichkeit an, wenn bestimmt wird, dass das Unionsrecht dann Vorrang vor dem Recht der Mitgliedstaaten genießt, wenn es ,in Ausübung der der Union übertragenen Zuständigkeiten“ erlassen ist. Das Bundesverfassungsgericht hat zu dieser Frage im Maastricht-Urteil eine sehr klare Aussage getroffen. „Würden“, so heißt es dort, „europäische Einrichtungen oder Organe den Unions-Vertrag in einer Weise handhaben oder fortbilden, die von dem Vertrag, wie er dem deutschen Zustimmungsgesetz zugrundeliegt, nicht mehr gedeckt wäre, so wären die daraus hervorgehenden Rechtsakte im deutschen Hoheitsbereich nicht verbindlich. Die deutschen Staatsorgane wären aus verfassungsrechtlichen Gründen gehindert, diese Rechtsakte in Deutschland anzuwenden. Dementsprechend prüft das Bundesverfassungsgericht, ob Rechtsakte der europäischen Einrichtungen und Organe sich in den Grenzen der ihnen eingeräumten Hoheitsrechte halten oder aus ihnen ausbrechen" 15 .

Der Vorbehalt des Bundesverfassungsgerichts, „ausbrechende“ Rechtsakte für in Deutschland unverbindlich zu erklären, und die Inanspruchnahme einer prinzipiellen Prüfungskompetenz stehen im Widerspruch zur Rechtsprechung des Europäischen Gerichtshofs, der für sich die vorbehaltlose Befugnis zur Letztentscheidung über die Auslegung und Anwendung des Gemeinschaftsrechts in Anspruch nimmt. Dieser Gegensatz beruht im Kern darauf, dass beide Gerichte ihre Positionen von je unterschiedlichen Ausgangspunkten her entwickeln. Von ihren jeweiligen Prämissen aus betrachtet sind beide Positionen in sich schlüssig, untereinander jedoch sind sie unvereinbar.

Das Bundesverfassungsgericht betont, dass Deutschland ,auch nach dem Inkrafttreten des Unions-Vertrags Mitglied in einem Staatenverbund“ ist, „dessen Gemeinschaftsgewalt sich von den Mitgliedstaaten ableitet und im deutschen Hoheitsbereich nur kraft des deutschen Rechtsanwendungsbefehls verbindlich wirken kann. Geltung und Anwendung von Europarecht in Deutschland hängen von dem Rechtsanwendungsbefehl des Zustimmungsgesetzes ab“16. Der Rechtsanwendungsbefehl des Zustimmungsgesetzes zu dem völkerrechtlichen Vertrag, mit dem Hoheitsrechte auf die Gemeinschaft bzw. auf die Union übertragen werden, ist der „Hebel“, mit dem sich das Bundesverfassungsgericht die prinzipielle Prüfungsbefugnis über das Gemeinschaftsrecht eröffnet. Der Europäische Gerichtshof „kappt“ dagegen diese Verbindung. Die Mitgliedstaaten hätten das Gemeinschaftsrecht als eine eigenständige Rechtsordnung mit autonomer Gemeinschaftsgewalt geschaffen. Aus der damit verbundenen Loslösung der Gemeinschaft von ihren völkerrechtlichen und nationalverfassungsrechtlichen

14 Vgl. BVerfGE 89, S. 155 (182ff.).

15 BVerfGE 89, S. 155 (188).

16 BVerfGE 89, S. 155 (190). 
Grundlagen folge nicht nur der Vorrang des Gemeinschaftsrechts vor dem nationalen Recht. Diese Autonomie impliziere vielmehr auch, dass gemeinschaftliche Rechtsakte nicht von nationalen Gerichten verworfen werden könnten.

Betonen möchte ich freilich, dass das praktische Konfliktpotential zwischen Bundesverfassungsgericht und Europäischem Gerichtshof auch hinsichtlich der Einhaltung der Kompetenzgrenzen der Gemeinschaft nicht überbewertet werden sollte. Die gegenwärtige Rechtsprechung beider Gerichte wird also nicht vom „Ernstfall“ eines Souveränitätskonflikts geprägt, sondern stellt sich viel eher als eine - im positiven Sinne - konkurrierende Tätigkeit im Prozess der fortschreitenden europäischen Integration durch Recht dar.

\section{Die Mangold-Entscheidung des EuGH}

Lassen Sie mich abschließend noch kurz eine im Zweiten Senat des Bundesverfassungsgerichts aus dem Bereich des Arbeitsrechts anhängige Verfassungsbeschwerde ansprechen, die im Zusammenhang mit der bekannten Mangold-Entscheidung des Europäischen Gerichtshofs vom 22. November $2005^{17}$ zur Befristung von Arbeitsverträgen steht. Wie Sie wissen, ist diese Entscheidung, die das Verbot der Diskriminierung wegen des Alters als einen allgemeinen Grundsatz des Gemeinschaftsrechts ansieht, nicht zuletzt unter dem Gesichtspunkt der Kompetenz des Gerichtshofs bzw. der Gemeinschaft in der Literatur durchaus auch auf Kritik gestoßen ${ }^{18}$. Ein Urteil des Bundesarbeitsgerichts vom 26. Mai $2006^{19}$ zur wirksamen Befristung eines Arbeitsverhältnisses folgt demgegenüber der Rechtsprechung des Europäischen Gerichtshofs und stellt fest, der Gerichtshof habe mit der Entscheidung nicht die mit den deutschen Zustimmungsgesetzen auf die Gemeinschaft übertragenen Kompetenzen überschritten.

Gegen dieses Urteil wendet sich der beklagte Arbeitgeber mittels der Verfassungsbeschwerde 20 und trägt zur Begründung unter anderem vor, dass es sich bei der Mangold-Entscheidung um einen ausbrechenden Rechtsakt des Europäischen Gerichtshofs handele.

17 EuGH NJW 2005, S. 3695ff.

18 So titelte etwa Bauer, NZA 2005, S. 800ff. „Ein Stück aus dem Tollhaus: Altersbefristung und der EuGH“.

19 BAG NZA 2006, S. $1162 \mathrm{ff}$.

202 BvR 2661/06. 


\section{Diskussionsbericht zu Teil I}

\section{Diskussionsleitung: Pauliine KOSKELO}

Im Anschluss an den Vortrag von Papier wurde unter Bezugnahme auf die Einführung Münteferings und von Maydells Referat nochmals betont, dass das Gemeinschaftsrecht viele Regelungen im Bereich der Finanz- und Wirtschaftspolitik trifft, während Sozial- und Arbeitsrecht größtenteils Sache des nationalen Gesetzgebers sind. Dieses Ungleichgewicht führe auf europäischer Ebene zu einer schwachen Position sozialer Rechte, gerade auch in gerichtlichen Verfahren, und mache es für Gewerkschaften auf europäischer Ebene schwer, als politisches Gegengewicht auf die zunehmende Öffnung der Märkte zu reagieren. So seien gerade die für Gewerkschaften so wichtigen Bestimmungen über Arbeitskampfmaßnahmen ausschließlich auf nationaler Ebene geregelt. Daher wurde Präsident Skouris gefragt, ob sich der EuGH aufgrund der Kompetenzverteilung im Vertrag von Amsterdam daran gehindert sieht, dem eben geschilderten Ungleichgewicht von Finanz- und Wirtschaftspolitik auf der einen und Sozial- und Arbeitsrecht auf der anderen Seite etwas entgegenzusetzen und ob die auf dem Brüsseler Gipfel im Juni 2007 beschlossene Einbeziehung der Grundrechtecharta hier eine Verbesserung bedeute. Skouris erwiderte darauf, dass er sich bei der Beurteilung von solchen Entwicklungen des Gemeinschaftsrechts eine gewisse Vorsicht angeeignet habe, gerade auch eingedenk des Schicksals des Europäischen Verfassungsvertrags, der im Oktober 2004 von allen Regierungschefs in Rom feierlich unterschrieben wurde. Das bisherige Nichtinkrafttreten der Charta habe jedoch den EuGH nicht daran gehindert, seine gut funktionierende Rechtsprechung zum Arbeits- und Sozialrecht kontinuierlich fortzuführen und fortzuentwickeln, was zahlreiche Beispiele aus den vergangenen 50 Jahren zeigten. Eine verbindliche Geltung der Charta würde aber dennoch einen immensen Fortschritt bedeuten, da es im Interesse eines jeden Richters sei, auf der Grundlage von klaren und fundierten Rechtsquellen entscheiden zu können. In diesem Zusammenhang bemerkte Skouris noch zur viel kritisierten Rechtsprechung des EuGH im Fall Mangold, dass der Gerichtshof diesen Fall auf Grundlage eindeutiger Rechtsquellen entschieden habe. Denn die Frage fehlender Diskriminierung oder Nichtdiskriminierung wegen Alters sei keine Erfindung des Gerichtshofs, sondern eine des Gesetzgebers, die der EuGH dann nur konsequent angewendet habe. Generell dürfe sich der Gesetzgeber nicht darüber wundern, wenn von ihm beschlossene Regelungen von den Gerichten auch angewendet würden.

Im Anschluss an diese pointierte Bemerkung über das Verhältnis von Gesetzgebung und Rechtsprechung wurde festgestellt, dass 50 Jahre Europäische Gemeinschaft auch 50 Jahre der Multilateralisierung des internationalen Sozialrechts bedeuteten. So regelte schon die VO EWG/3/58 als einer der ersten Rechtsetzungsakte der Europäischen Ge- 